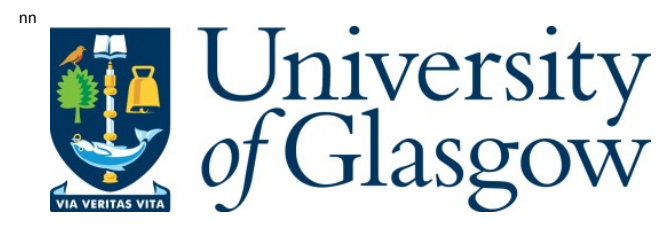

M oodie, E. E.M ., Dean, N ., and Sun, Y . R . (2014) Q-learning: flexible learning about useful utilities. Statistics in Biosciences, 6 (2). pp. 223-243.

Copyright @ 2014 Springer

A copy can be downloaded for personal non-commercial research or study, without prior permission or charge

Content must not be changed in any way or reproduced in any format or medium without the formal permission of the copyright holder(s)

http://eprints.gla.ac.uk/85869/

Deposited on: 30 J anuary 2015

Enlighten - Research publications by members of the University of Glasgow http://eprints.gla.ac.uk 


\title{
Q-learning: Flexible learning about useful utilities
}

\author{
Erica E. M. Moodie ${ }^{* 1}$, Nema Dean ${ }^{\dagger 2}$, and Yue Ru $\mathrm{Sun}^{3}$ \\ ${ }^{1}$ Department of Epidemiology, Biostatistics, and Occupational Health, McGill University \\ ${ }^{2}$ School of Mathematics and Statistics, University of Glasgow \\ ${ }^{3}$ Department of Mathematics and Statistics, School of Computer Science, McGill University
}

\section{August 2013}

\begin{abstract}
Dynamic treatment regimes are fast becoming an important part of medicine, with the corresponding change in emphasis from treatment of the disease to treatment of the individual patient. Because of the limited number of trials to evaluate personally tailored treatment sequences, inferring optimal treatment regimes from observational data has increased importance. Q-learning is a popular method for estimating the optimal treatment regime, originally in randomized trials but more recently also in observational data. Previous applications of Q-learning have largely been restricted to continuous utility end-points with linear relationships. This paper is the first both to extend the framework to discrete utilities and to implement the modelling of covariates from linear to more flexible modelling using the generalized additive model (GAM) framework. Simulated data results show that the GAM adapted Q-learning typically outperforms Q-learning with linear models and other frequently-used methods based on propensity scores in terms of coverage and bias/MSE. This represents a promising step towards a more fully general Q-learning approach to estimating optimal dynamic treatment regimes.

This article is in technical report form, the final publication is available at http://www.springerlink.com/openurl.asp?genre=article\&id=doi:10.1007/s12561-0139103-Z

Keywords:Dynamic Treatment Regimes, Q-learning, Generalized Additive Models, Discrete data
\end{abstract}

\section{Introduction}

The call for treatments that are personalized to the individual is growing more urgent, particularly as the population ages and care for chronic diseases becomes increasingly necessary. For example, Topol (27) writes that we have entered "a new era of medicine, in which each person can be near fully defined at the individual level, instead of how we practice medicine at the population level". He further goes on to discourage "use of the same medication and dosage for a diagnosis rather than for a patient." While

\footnotetext{
*erica.moodie@mcgill.ca

†Nema.Dean@glasgow.ac.uk
} 
it is true that high-dimensional data, even genome scans, are increasingly available to the average "consumer" of medicine, there remains relatively few studies which provide rigorous evaluations of tailored approaches to treatment, also called dynamic treatment regimes (DTRs). There has been a number of sequentially randomized trials designed to evaluate personally-tailored sequences of treatment $(21,5,25)$, however these trials are often expensive and require large samples. Estimating DTRs from observational data is therefore important.

In such studies, an optimal DTR is sought, where optimality is with respect to some outcome which may represent some end-of-study measure such as 12-month symptom score (22) or a cumulative measure such as percent of time in the study that a biological marker remains in the target range (20), or even a composite utility summarizing both effectiveness and tolerability (26). Given the complexities and potential highdimensionality involved in the longitudinal distribution of a sequence of covariates, exposures, and the outcome, semi-parametric estimation procedures have dominated the statistical literature on DTRs. Q-learning (24; 14) is a particularly appealing approach which has grown in popularity (e.g. 32, 4, 33, 1, 11, 15; 23) in the last half-decade due to the ease with which it can be implemented and, perhaps more importantly, understood by non-statistical collaborators.

In the DTR literature, Q-learning has principally been used in the context of randomized trials. However, it has recently been shown that Q-learning can be used for observational data, provided models for Q-functions, which we will define forthwith, can be correctly specified. Correct specification of the Q-functions can be challenging; employing a flexible model is appealing, but must be done with care as the number of parameters to be estimated in a DTR setting may already be large in an observational data setting with many intervals of treatment, and multiple covariates at each interval.

Generalized additive models (GAMs), as implemented in the mgcv package (28, 30) in the R statistical software language (16) are a useful approach to allowing greater flexibility in modeling the relationship between outcome and different covariates. In this approach, GAMs are treated as penalized regression splines with different smoothing parameters allowed for each covariate. Automatic selection of the smoothing parameters is performed through generalized cross-validation. This allows for more parsimonious smoothing model selection, keeping the number of parameters to a minimum, which is important given the potentially large covariate space in the DTR setting.

Another significant challenge arises when the outcome of interest is a discrete-valued utility or loss function. These types of utilities commonly arise in a variety of contexts. For example, in the context of a cohort of HIV-hepatitis coinfected individuals, the utility could be the number of emergency room admissions in a six-month period, or even the event of initiating hepatitis $\mathrm{C}$ therapy, where the treatment regime of interest leading to such an outcome may be a drug or alcohol addiction program. To date, Q-learning has been considered primarily for continuous valued outcomes such as symptom scores $(13$; 4,$11 ; 3)$ and, less commonly, censored time-to-event data (33, 9).

In this paper, we demonstrate the use of Q-learning using generalized additive models with penalized regression splines selected via generalized cross validation (28, 30) for continuous and discrete outcomes. In the next section, we introduce Q-learning and then proceed to describe the generalization of the usual Q-learning procedure for continuous outcomes to its discrete-outcome counterpart. Next, we provide a brief background to generalized additive models and explain how they may be used to add flexibility into the modelling stages of the Q-learning procedure. In Section 3, we simulate from a wide 
variety of settings to assess the performance of the continuous and the discrete-utility Q-learning algorithm with flexible-modelling. Section 4 discusses the various aspects of the approach and concludes with caveats about the method in general and comparisons to alternative approaches.

\section{Q-learning}

Our development will focus on the two-interval setting, however any finite number of treatment intervals could be considered. Longitudinal data on a single patient are given by the trajectory $\left(C_{1}, O_{1}, A_{1}, C_{2}, O_{2}, A_{2}, Y\right)$, where $C_{j}$ and $O_{j}(j=1,2)$ denote the covariates measured prior to treatment at the beginning of the $j$-th interval, $A_{j}(j=1,2)$ is the treatment assigned at the $j$-th interval subsequent to observing $O_{j}$, and $Y$ is a utility measured at the end of the second interval (where larger values are preferred). We distinguish two types of covariates: those variables $O_{j}$ that interact with treatment, called tailoring or prescriptive variables, and those variables $C_{j}$ that do not interact with treatment, called predictive variables. For the purposes of this paper, we shall assume that the prescriptive variables, $O_{j}$, are not confounding variables, whereas all predictive variables, $C_{j}$, are potentially so. The history at each interval is denoted as $H_{1}=\left(C_{1}, O_{1}\right), H_{2}=\left(C_{1}, O_{1}, A_{1}, C_{2}, O_{2}\right)$.

We consider studies in which there are two possible treatments at each interval, $A_{j} \in$ $\{-1,1\}$, and the receipt of treatment $A_{j}$ may depend on the observed value of covariates $C_{j}$ and $O_{j}$, but not on any unmeasured variables. A two-interval DTR consists of two decision rules, $\left(d_{1}, d_{2}\right)$, with $d_{j} \equiv d_{j}\left(H_{j}\right) \in \mathcal{A}_{j}$, where $\mathcal{A}_{j}$ is the set of possible treatments at the $j$-th interval.

\subsection{Basic Q-learning for a continuous utility}

Q-learning is closely related to finding a dynamic program, in that estimation begins at the last interval, and the optimal treatment at each interval is then found estimating the impact of treatment in that interval on a "pseudo-outcome" which is constructed by assuming all subsequent treatments are optimal; when the outcome is continuous, the pseudo-outcome can be interpreted as the predicted counterfactual outcome under optimal treatment in future intervals.

Define the $Q$-functions for the two intervals as follows:

$$
\begin{aligned}
& Q_{2}\left(H_{2}, A_{2}\right)=E\left[Y \mid H_{2}, A_{2}\right] \\
& Q_{1}\left(H_{1}, A_{1}\right)=E\left[\max _{a_{2}} Q_{2}\left(H_{2}, a_{2}\right) \mid H_{1}, A_{1}\right] .
\end{aligned}
$$

If the outcome was continuous, a typical modelling choice for the Q-functions would be to use linear models

$$
Q_{j}\left(H_{j}, A_{j} ; \beta_{j}, \psi_{j}\right)=\beta_{j}^{T} H_{j 0}+\left(\psi_{j}^{T} H_{j 1}\right) A_{j},
$$

at the $j$-th interval $(j=1,2)$ where $H_{j 0}$ and $H_{j 1}$ are two (possibly different) summaries of the history $H_{j}$. We use $H_{j 0}$ to contain the collection of variables that have a predictive effect on the outcome that is not modified by the treatment, and $H_{j 1}$ to denote the components of the history that do interact with treatment. Both $H_{j 0}$ and $H_{j 1}$ include a constant, or intercept, term. 
Q-learning proceeds by a series of regressions. First, a linear model such as Equation (1) is fit to estimate parameters of the last-interval Q-function, $Q_{2}$, often using ordinary least squares or some other standard form of regression. Using the parameter estimates from the last interval, a pseudo-outcome is formed by taking

$$
\widetilde{Y}_{j i}=\max _{a_{2}} Q_{2}\left(H_{2 i}, a_{2} ; \hat{\beta}_{2}, \hat{\psi}_{2}\right)=\hat{\beta}_{2}^{T} H_{20, i}+\left|\hat{\psi}_{2}^{T} H_{21, i}\right| .
$$

The pseudo-outcome represents an estimate of the expected outcome for an individual with treatment and covariate history $H_{2 i}=h_{2}$ were they treated optimally at the final interval. That is, the pseudo-outcome $\widetilde{Y}_{j i}$ is a predictor of the unobserved random variable $\max _{a_{2}} Q_{2}^{o p t}\left(H_{2 i}, a_{2}\right), i=1, \ldots, n$. This pseudo-outcome is then used as the response which is then modelled linearly (again, using a form such as that in Equation (1)) with estimation performed via OLS.

Once the Q-functions have been estimated, finding the optimal decision rules is straightforward. The estimated optimal DTR is simply given by

$$
\hat{d}_{j}^{o p t}\left(h_{j}\right)=\arg \max _{a_{j}} Q_{j}^{o p t}\left(h_{j}, a_{j} ; \hat{\beta}_{j}, \hat{\psi}_{j}\right), j=1,2 .
$$

Q-learning is conceptually quite simple to understand and put into practice, and has been implemented in the R (16) package qLearn (31) which is available from the Comprehensive R Archive Network (CRAN) at http://cran.r-project.org/web/packages/qLearn/index.html.

Q-learning is not restricted to two intervals, but can be applied in any setting where there is interest in estimating the optimal DTR over a fixed set of treatment intervals. If we let $Q_{K+1}^{o p t} \equiv 0$, and

$$
Q_{j}^{o p t}\left(H_{j}, A_{j}\right)=E\left[\max _{a_{j+1}} Q_{j+1}^{o p t}\left(H_{j+1}, a_{j+1}\right) \mid H_{j}, A_{j}\right], \quad j=1, \ldots, K,
$$

we can again assume a linear parameterization of the $j$ th interval Q-function as follows:

$$
Q_{j}^{o p t}\left(H_{j}, A_{j} ; \beta_{j}, \psi_{j}\right)=\beta_{j}^{T} H_{j 0}+\left(\psi_{j}^{T} H_{j 1}\right) A_{j}, \quad j=1, \ldots, K .
$$

Then starting at the $K$ th interval and moving backward through each interval, the estimates $\left(\hat{\beta}_{j}, \hat{\psi}_{j}\right)$ of the regression parameters can be estimated using

$$
\arg \min _{\beta_{j}, \psi_{j}} \frac{1}{n} \sum_{i=1}^{n}\left(\widetilde{Y}_{j i}-Q_{j}^{o p t}\left(H_{j i}, A_{j i} ; \beta_{j}, \psi_{j}\right)\right)^{2},
$$

where $\widetilde{Y}_{j i}=\max _{a_{j+1}} Q_{j+1}^{o p t}\left(H_{j+1}, a_{j+1} ; \hat{\beta}_{j+1}, \hat{\psi}_{j+1}\right)$ is the pseudo-outcome at the $j$ th interval, representing the expected outcome for an individual with treatment and covariate history $H_{j i}=h_{j}$ were they treated optimally from interval $j+1$ onwards. As in the twointerval setting, the estimated optimal DTR is defined to be the regime which maximizes the interval-specific

Q-functions:

$$
\hat{d}_{j}^{o p t}\left(h_{j}\right)=\arg \max _{a_{j}} Q_{j}^{o p t}\left(h_{j}, a_{j} ; \hat{\beta}_{j}, \hat{\psi}_{j}\right), j=1, \ldots, K .
$$

Q-learning may also be used where the utilities are measured at each interval by taking the pseudo-outcome at the $j$ th interval to be

$$
\widetilde{Y}_{j i}=Y_{j i}+\max _{a_{j+1}} Q_{j+1}^{o p t}\left(H_{j+1}, a_{j+1} ; \hat{\beta}_{j+1}, \hat{\psi}_{j+1}\right)
$$

where $Y_{j i}, j=1,2, \ldots, K$ are the interval-specific utilities (with $Y_{i} \equiv Y_{K i}$ ). 


\section{$2.2 \quad$ Q-learning for a binary utility}

If the utility function $Y$ is discrete, the modelling procedure of the previous section must be adapted. At the final interval, we have $Q_{2}\left(H_{2}, A_{2}\right)=E\left[Y \mid H_{2}, A_{2}\right]$. A reasonable modelling choice would be to consider a generalized linear model - e.g. for a Bernoulli utility, we might choose a logistic model of the form $E\left[Y \mid H_{2}, A_{2}\right]=\operatorname{expit}\left(\beta_{\mathrm{j}}^{\mathrm{T}} \mathrm{H}_{\mathrm{j} 0}+\left(\psi_{\mathrm{j}}^{\mathrm{T}} \mathrm{H}_{\mathrm{j} 1}\right) \mathrm{A}_{\mathrm{j}}\right)$, where $\operatorname{expit}(\mathrm{x})=\exp (\mathrm{x}) /(1+\exp (\mathrm{x}))$ is the inverse-logit function. Then

$$
Q_{1}\left(H_{1}, A_{1} ; \beta_{1}, \psi_{1}\right)=\max _{a_{2}} Q_{2}\left(H_{2 i}, a_{2} ; \hat{\beta}_{2}, \hat{\psi}_{2}\right)=\operatorname{expit}\left(\hat{\beta}_{2}^{\mathrm{T}} \mathrm{H}_{20, \mathrm{i}}+\left|\hat{\psi}_{2}^{\mathrm{T}} \mathrm{H}_{21, \mathrm{i}}\right|\right),
$$

which is bounded by $[0,1]$.

As in the continuous utility setting, the optimal regime at the first interval is defined by

$$
\hat{d}_{1}\left(h_{1}\right)=\arg \max _{a_{1}} Q_{1}\left(h_{1}, a_{1} ; \hat{\beta}_{1}, \hat{\psi}_{1}\right) .
$$

However, assuming the model expit $\left(\beta_{1}^{\mathrm{T}} \mathrm{H}_{10}+\left(\psi_{1}^{\mathrm{T}} \mathrm{H}_{11}\right) \mathrm{A}_{1}\right)$ for $Q_{1}\left(H_{1}, A_{1} ; \beta_{1}, \psi_{1}\right)$, we see that the optimal first-interval treatment can be obtained by maximizing expit $\left(\beta_{1}^{\mathrm{T}} \mathrm{H}_{10}+\left(\psi_{1}^{\mathrm{T}} \mathrm{H}_{11}\right) \mathrm{A}_{1}\right)$ or by simply maximizing $\beta_{1}^{T} H_{10}+\left(\psi_{1}^{T} H_{11}\right) A_{1}$, since the inverselogit function is strictly increasing. That is, rather than modelling the predicted probability of success in the second interval under the optimal DTR, expit $\left(\hat{\beta}_{2}^{\mathrm{T}} \mathrm{H}_{20, \mathrm{i}}+\left|\hat{\psi}_{2}^{\mathrm{T}} \mathrm{H}_{21, \mathrm{i}}\right|\right)$, which is bounded in $[0,1]$, we model the logit of that probability, $\hat{\beta}_{2}^{T} H_{20, i}+\left|\hat{\psi}_{2}^{T} H_{21, i}\right|$, which takes values in the real line. This allows us to use ordinary least squares to estimate parameters in a model for the logit of $Q_{1}\left(h_{1}, a_{1} ; \hat{\beta}_{1}, \hat{\psi}_{1}\right)$.

The Q-learning algorithm for binary outcomes consists of the following steps:

1. Interval 2 parameter estimation: Using logistic regression, find estimates $\left(\hat{\beta}_{2}, \hat{\psi}_{2}\right)$ of the conditional mean model for $Y$ (i.e. the conditional probability of a success), $Q_{2}\left(H_{2 i}, A_{2 i} ; \beta_{2}, \psi_{2}\right)$.

2. Interval 2 optimal rule: Set $\hat{d}_{2}\left(h_{2}\right)=\arg \max _{a_{2}} Q_{2}\left(h_{2}, a_{2} ; \hat{\beta}_{2}, \hat{\psi}_{2}\right)$.

3. Interval 1 pseudo-outcome: Set

$$
\widetilde{Y}_{1 i}=\max _{a_{2}} \operatorname{logit}\left(Q_{2}\left(H_{2 i}, a_{2} ; \hat{\beta}_{2}, \hat{\psi}_{2}\right)\right), i=1, \ldots, n .
$$

4. Interval 1 parameter estimation: Using ordinary least squares regression, find estimates

$$
\left(\hat{\beta}_{1}, \hat{\psi}_{1}\right)=\arg \min _{\beta_{1}, \psi_{1}} \frac{1}{n} \sum_{i=1}^{n}\left(\widetilde{Y}_{1 i}-Q_{1}\left(H_{1 i}, A_{1 i} ; \beta_{1}, \psi_{1}\right)\right)^{2} .
$$

5. Interval 1 optimal rule: Set $\hat{d}_{1}\left(h_{1}\right)=\arg \max _{a_{1}} Q_{1}\left(h_{1}, a_{1} ; \hat{\beta}_{1}, \hat{\psi}_{1}\right)$.

The estimated optimal DTR using Q-learning is given by $\left(\hat{d}_{1}, \hat{d}_{2}\right)$. Note that unlike in the continuous utility setting, for the pseudo-outcome, $\widetilde{Y}_{1 i}$, in a binary utility scenario does not represent the (expected) value of the second-interval Q-function under the optimal treatment but rather a transformation of that expected outcome. 


\subsection{Q-learning for an unbounded, discrete-valued utility}

If, rather than a binary-valued variable, the utility were defined in terms of a count, the above-stated procedure could be adapted at Steps 1, 3, and 5:

1. Interval 2 parameter estimation: Using Poisson regression, find estimates $\left(\hat{\beta}_{2}, \hat{\psi}_{2}\right)$ of the conditional mean model for $Y$ (i.e. the conditional mean utility), $Q_{2}\left(H_{2 i}, A_{2 i} ; \beta_{2}, \psi_{2}\right)$.

2. Interval 2 optimal rule: Set $\hat{d}_{2}\left(h_{2}\right)=\arg \max _{a_{2}} Q_{2}\left(h_{2}, a_{2} ; \hat{\beta}_{2}, \hat{\psi}_{2}\right)$.

3. Interval 1 pseudo-outcome: Set

$$
\widetilde{Y}_{1 i}=\max _{a_{2}} \log \left(Q_{2}\left(H_{2 i}, a_{2} ; \hat{\beta}_{2}, \hat{\psi}_{2}\right)\right), i=1, \ldots, n .
$$

4. Interval 1 parameter estimation: Using ordinary least squares regression, find estimates $\left(\hat{\beta}_{1}, \hat{\psi}_{1}\right)$ of the conditional mean model for $\widetilde{Y}_{1 i}$ (i.e. the pseudo-outcome), $Q_{1}\left(H_{2 i}, A_{2 i} ; \beta_{2}, \psi_{2}\right)$.

5. Interval 1 optimal rule: Set $\hat{d}_{1}\left(h_{1}\right)=\arg \max _{a_{1}} Q_{1}\left(h_{1}, a_{1} ; \hat{\beta}_{1}, \hat{\psi}_{1}\right)$.

The estimated optimal DTR using Q-learning is given by $\left(\hat{d}_{1}, \hat{d}_{2}\right)$. Thus the approach closely mimics that taken for the Bernoulli utility, where the pseudo-outcome is defined to be the maximum of the linear predictor in the Step 1 regression under optimal treatment. An alternative strategy could be adopted in Steps 3 and 4: rather than using OLS with the pseudo-outcome taken as the largest value of linear predictor for each individual, a generalized linear model with a log-link could be employed, defining the pseudo-outcome to be the expected count under optimal second-interval treatment. In adopting the alternative strategy, the pseudo-outcome has a more natural interpretation, much as in the continuous data setting: in the discrete count utility scenario, it can be seen to represent the (expected) conditional utility under the optimal treatment at the second interval. However, the approach of using a GLM for both the first and second interval estimates comes at a significant computational cost, as the GAM fitting procedure with a Poisson family specification is noticeably slower than its Gaussian family counterpart.

\subsection{Adding flexibility to the Q-function}

The standard form of input for covariates in the Q-function model is additive and linear. While these models allow ease of interpretation and simplicity of implementation, they may lack the flexibility to adequately capture more complex relationships between the outcome and prescriptive and predictive variables. Indeed, often there is no reason (other than pragmatism) to believe the form of the relationship between outcome variable and explanatory variables will be linear.

A generalized additive model (GAM) is a semi-parametric extension of both linear and generalized linear models first introduced by Hastie (7, 8, 29). The model is still additive but each explanatory variable's relationship to the outcome variable is given by a smooth function of the explanatory variable.

$$
E\left[Q_{j} \mid X\right]=\mathbf{X} \beta+s_{1}\left(X_{1}\right)+s_{2}\left(X_{2}\right)+s_{3}\left(X_{3}, X_{4}\right)+s_{4}\left(X_{5}\right)+\cdots
$$

Here, $\mathbf{X}$ represents the design matrix made up of variables that enter the model in standard linear form (including the intercept), while the Q-function also depends on some or all of the variables $X_{j}$ through unknown smooth functions $s_{j}$. 
The most commonly used smooth functions, $s_{j}$, are penalized regression splines. In order to avoid overfitting, a penalty (usually of the form of the integral of the squared second derivative of the smooth function multiplied by a smoothness parameter) is applied for each of the smoothing functions and the resulting penalized log-likelihood, given by Equation (2) is maximized.

$$
l(\eta)-\frac{1}{2} \sum_{j} \theta_{j} \int\left[s_{j}^{\prime \prime}(x)\right]^{2} d x
$$

where $l$ is the log-likelihood of the linear predictor. This penalty aims to punish smooth functions that are too "wiggly". There is a separate smoothing parameter $\theta_{j}$ for each $s_{j}$, as different covariates may require different levels of smoothing.

The size of smoothing parameters must be controlled in order to avoid over-fitting and decreased efficiency; this can be achieved using generalized cross-validation (6). Generalized cross-validation (GCV) is a weighted version of ordinary cross-validation where the GCV score for a model with smoothing parameter vector value $\theta$ is the average residual sum of squares for the model, weighted by $\frac{1}{(1-\operatorname{tr} H(\theta) / n)^{2}}$, where $H(\theta)$ is the hat matrix for the smoothing model fit using smoothing parameter values $\theta$. This score can be computed easily for different $\theta$ 's and the final $\theta$ used is the one with the smallest GCV score. The GCV is approximately the same as the $C_{p}$ (Unbiased Risk Method) criterion. GCV was originally proposed as a computationally cheaper way of selecting penalty parameters but it has several favorable properties (6, 10). While the GCV has non-zero probability of selecting 0 for the smoothing parameter values (i.e. no smoothing), this probability tends to zero exponentially fast with increasing sample size.

If the true underlying function is linear, the selected smoothing parameters should reflect this, as well as being able to capture more complex underlying functions.

\section{Simulations}

In this section, we consider a simulation study to compare the performance of a GAM for the Q-function as well as a variety of other approaches to covariate adjustment. In particular, we contrast no covariate adjustment with adjustment by including covariates as linear terms in the Q-function; including the propensity score (PS) directly in the Q-function, including quintiles of the PS as covariates in the Q-function, and inverse probability of treatment weighting (IPTW) (further detail on these methods is given in section 3.2). In addition to assessing the bias and variability of the point estimates, percentile bootstrap confidence intervals were used to assess coverage rates of $95 \%$ confidence intervals. The generative model and the analysis model are straightforward adaptations of the corresponding models described in (4) and (11).

\subsection{Generative models}

\subsubsection{Continuous utilities}

We consider a single continuous covariate, $C_{j}$, at each interval, where $C_{1} \sim \mathcal{N}(0,1)$ and $C_{2} \sim \mathcal{N}\left(\eta_{0}+\eta_{1} C_{1}, 1\right)$ for $\eta_{0}=-0.5, \eta_{1}=0.5$. Treatment assignment is dependent on the value of $C_{j}: P\left[A_{j}=1 \mid C_{j}\right]=1-P\left[A_{j}=-1 \mid C_{j}\right]=\operatorname{expit}\left(\zeta_{0}+\zeta_{1} C_{j}\right), j=1,2$ where $\operatorname{expit}(x)=\exp (x) /(1+\exp (x))$ and $\zeta_{0}=-0.8, \zeta_{1}=1.25$. The binary tailoring variables 
were generated via $P\left[O_{1}=1\right]=\frac{1}{2}$ and $P\left[O_{2}=1 \mid O_{1}, A_{1}\right]=\operatorname{expit}\left(\delta_{1} O_{1}+\delta_{2} A_{1}\right)$. Let $\mu=E\left[Y \mid C_{1}, O_{1}, A_{1}, C_{2}, O_{2}, A_{2}\right]$, and $\epsilon \sim N(0,0.25)$ be the error term. Then $Y=\mu+\epsilon$, where

$$
\begin{gathered}
\mu=\gamma_{0}+\gamma_{1} C_{1}+\gamma_{2} O_{1}+\gamma_{3} A_{1}+\gamma_{4} O_{1} A_{1}+\gamma_{5} C_{2}+\gamma_{6} A_{2}+\gamma_{7} O_{2} A_{2}+ \\
\gamma_{8} A_{1} A_{2}+f_{1}\left(C_{1}\right)+f_{2}\left(C_{2}\right) .
\end{gathered}
$$

We considered only "regular" data-generating scenarios, i.e. scenarios in which there the Q-learning estimators are consistent and asymptotically Normal; for this reason, only the usual Q-learning estimator was implemented, rather than recent variants which have been proposed for non-regular settings (i.e. those under which the Q-learning estimator is biased, and asymptotically non-Normal) (12; 4; 23). Hence, we focused on settings where $\gamma=(0,1,0,-0.5,0,1,0.25,0.5,0.5)$ and $\delta=(0.1,0.1)$.

We first considered a simple linear relationship between the confounders $C_{1}, C_{2}$ and the outcome, taking $f_{1}\left(C_{1}\right)=0$ and $f_{2}\left(C_{2}\right)=0$. In two additional settings, we added non-linearity to the relationship using

$$
f_{1}\left(C_{1}\right)=5 C_{1}^{2}, f_{2}\left(C_{2}\right)=3 C_{2}^{2}
$$

and then

$$
f_{1}\left(C_{1}\right)=5 C_{1}^{2}, f_{2}\left(C_{2}\right)=4 C_{2}^{2} \mathbb{I}\left[C_{2}<-1\right]+C_{2}^{3} \mathbb{I}\left[C_{2}>.25\right] .
$$

In previous work (11), we considered also simulations where $\mu$ was a function of only treatments and tailoring variables so as to evaluate the ability of a variety of methods of adjustment in settings where all Q-function models were correctly specified. We do not pursue this approach here, as we considered the setting to be rather too artificial to reflect real data analysis practices where covariates do in fact affect the mean response.

\subsubsection{Discrete utilities}

For discrete utilities measured only in the final stage, much of the data-generation proceeded as in the continuous utility case. Derivations of the true decision rule parameters as a function of the data-generating parameters can be found in the Appendix.

In both discrete utility settings, $C_{j}$ and $O_{j}$ were determined in the same way as in the continuous utility case, as detailed in Section 3.1.1. For the Bernoulli utility, we set

$$
\begin{aligned}
\mu= & E\left[Y \mid C_{1}, O_{1}, A_{1}, C_{2}, O_{2}, A_{2} ; \gamma\right] \\
= & \operatorname{expit}\left(\gamma_{0}+\gamma_{1} C_{1}+\gamma_{2} O_{1}+\gamma_{3} A_{1}+\gamma_{4} O_{1} A_{1}+\gamma_{5} C_{2}+\gamma_{6} A_{2}+\right. \\
& \left.\gamma_{7} O_{2} A_{2}+\gamma_{8} A_{1} A_{2}+f_{1}\left(C_{1}\right)+f_{2}\left(C_{2}\right)\right) .
\end{aligned}
$$

Thus $P\left[Y=1 \mid C_{1}, O_{1}, A_{1}, C_{2}, O_{2}, A_{2} ; \gamma\right]=\mu$. As in the continuous utility case, we avoided nonregular scenarios, setting $\gamma=(0,1,0,-0.5,0,1,0.25,0.5,0.5)$ and $\delta=(0.1,0.1)$. We considered two different relationships between the confounders $C_{1}, C_{2}$ and the outcome. First, a linear relationship was considered by taking $f_{1}\left(C_{1}\right)=f_{2}\left(C_{2}\right)=0$. We next considered a quadratic confounder-outcome relationship, setting $f_{1}\left(C_{1}\right)=-1.2 C_{1}^{2}$ and $f_{2}\left(C_{2}\right)=1.4 C_{2}^{2}$.

In the case of a Poisson utility, we set

$$
\begin{gathered}
\mu=\exp \left(\gamma_{0}+\gamma_{1} C_{1}+\gamma_{2} O_{1}+\gamma_{3} A_{1}+\gamma_{4} O_{1} A_{1}+\gamma_{5} C_{2}+\gamma_{6} A_{2}+\right. \\
\left.\gamma_{7} O_{2} A_{2}+\gamma_{8} A_{1} A_{2}+f_{1}\left(C_{1}\right)+f_{2}\left(C_{2}\right)\right) .
\end{gathered}
$$

Thus $E\left[Y \mid C_{1}, O_{1}, A_{1}, C_{2}, O_{2}, A_{2} ; \gamma\right]=\mu$. We again took $\gamma=(0,1,0,-0.5,0,1,0.25,0.5,0.5)$ and $\delta=(0.1,0.1)$, and considered a linear $\left(f_{1}\left(C_{1}\right)=f_{2}\left(C_{2}\right)=0\right)$ and a quadratic $\left(f_{1}\left(C_{1}\right)=-0.4 C_{1}^{2}, f_{2}\left(C_{2}\right)=-0.6 C_{2}^{2}\right)$ confounder-outcome relationship. 


\subsection{Analyses}

As we noted above, correct specification of the Q-function is essential to estimating truly optimal treatment regimes (11). This implies the need for appropriate control of confounding when in an observational (non-randomized exposure) setting. We will consider several simple methods of adjusting for potentially confounding variables, and compare these with a general additive modelling approach.

Along with a simple linear specification of the Q-function, estimation based on propensity score models were considered. The propensity score (19) is a model for the probability of receiving treatment - often a predicted probability resulting from a logistic regression of treatment on covariates; in particular, it is the coarsest summary of confounding variables that serves to provide balance between the treated and untreated members of a sample. It is often used for dimension reduction, as it provides a scalar-valued summary of a possibly high-dimensional vector of confounding variables. Formally, the propensity score is defined as

$$
\pi(x)=P(A=1 \mid X=x)
$$

where $A$ is a binary treatment and $X$ is a collection of measured covariates (19). The propensity score is said to "balance" the distribution of covariates $X$ so that treatment received is independent of $X$ given $\pi(x)$. If the balancing property is achieved, the propensity score may be used in a variety of ways to obtain unbiased estimates of the treatment effect in a point-treatment setting provided $X$ contains all confounding variables. For example, the propensity score (or some function thereof, such as indicator variables for quintiles) may be used as a covariate in a regression model, or as a criterion on which to match treated and untreated subjects. Within narrow strata defined by the propensity score, all subjects - whether treated or untreated - will be similar with respect to their propensity scores, and hence with respect to covariates used to construct the propensity scores (or at least with those covariates whose estimated coefficients are not near zero).

The propensity score may also be used to construct inverse probability of treatment weights which are then used to weight the sample in the estimation procedure of choice. Specifically, an unbiased treatment effect estimator can be found in a point-treatment setting by weighting all treated subjects by $\pi(x)^{-1}$ and all untreated subjects by $(1-\pi(x))^{-1}$ in a one-interval setting (17); the weighting serves to up- or down-weight subjects so as to create a weighted sample in which covariates do not predict treatment exposure status and therefore do not act as confounders. In the multi-interval setting, inverse probability of treatment weighting may be generalized to weight each subject by the probability of receiving the observed treatment sequence, multiplying the interval-specific inverse weights. The weighting creates a pseudo-sample in which treatment receipt is independent of variables $X$ included in the propensity score. Inverse probability of treatment weighting requires that the propensity scores be bounded away from 0 and 1 to ensure the resulting weights are well-defined.

For each data-generating scenario, we compare six means of estimating the Q-functions:

1. no adjustment for the variables $C_{1}$ and $C_{2}$;

2. inclusion of $C_{1}$ and $C_{2}$ as linear terms in $Q_{2}\left(H_{2}, A_{2} ; \beta_{2}, \psi_{2}\right)$, and of $C_{1}$ as a linear term in $Q_{1}\left(H_{1}, A_{1} ; \beta_{1}, \psi_{1}\right)$;

3. inclusion of the interval-specific propensity score as a linear term in the model for each interval's Q-function; 
4. inclusion of indicators corresponding to quintiles of the interval-specific propensity score in the model for each interval's Q-function;

5. no adjustment for the variables $C_{1}$ and $C_{2}$, but estimation performed using inverse probability of treatment weighting; and

6. inclusion of (separate) splines on $C_{1}$ and $C_{2}$ in $Q_{2}\left(H_{2}, A_{2} ; \beta_{2}, \psi_{2}\right)$, and splines on $C_{1}$ in $Q_{1}\left(H_{1}, A_{1} ; \beta_{1}, \psi_{1}\right)$ selected via generalized cross-validation, i.e. the fitting of a GAM at each interval.

We were interested in assessing both bias and variability of the decision rule parameters, and hypothesized that a completely linear specification of the Q-function model would perform best when the utility was defined by a linear specification of all covariates, and that the GAM would outperform other approaches when the utility's dependence on the covariates was not linear. In previous work in which only linear dependence for continuous utilities was considered, it has been shown that regressing on a function of the propensity score typically does not provide good estimates of the first-interval decision rule parameters while inverse weighting can perform well.

\subsection{Results}

In Tables 1 and 2, respectively, we present results for the continuous and discrete utility settings, considering bias, variability, and coverage. Each simulated setting was run 1000 times. Attention is restricted to the parameter $\psi_{10}$, the parameter in the analytic model for the first-interval Q-function defined in Equation (1) which corresponds to the main effect of the treatment $A_{1} ; \psi_{10}$ has been found to be more sensitive to bias than $\psi_{11}$ (4; 23). In Figures 1 and 2, we present an alternative view of the model performance: each panel shows the mean outcome in a new population of 10,000 individuals in which we applied the true optimal DTR (using the known parameters from the data-generating mechanism) as well as the mean outcome in the new population in which the estimated DTRs are applied; results are shown for $n=250$ only, as the figures from estimates based on sample sizes of 1000 are very similar. Note that the form of the optimal rule depends only on parameters from the estimated Q-function models associated with treatment (i.e. the parameter associated with main effect of treatment, and all parameters associated with interactions between the treatment and tailoring variables), and so the true DTR is independent of the specification of the "main effects terms" for covariates other than the exposure in the model for the Q-function.

For the continuous outcome setting, in the first panel of Table 1, we observe excellent performance of both the linear (correct) specification and the GAM specification of the Q-function when the true confounder-outcome relationship is linear: bias is minimal, and the more flexible GAM-based estimator of the decision rule parameter exhibits low variability even for the smaller sample size of 250, implying there is little cost for the additional flexibility. Both estimators have coverage that does not differ significantly from the nominal level of 95\%. Correspondingly, performance under the DTR estimated using the linear model and the GAM are essentially as good as under the truth (Figure 1, left panel); mean outcomes under the IPTW estimated regime are equally good.

When the dependence of the utility on the confounding variables is not linear (second and third panels of Table 1), only the estimator derived from a GAM for the Q-function exhibits unbiasedness, low variability, and good coverage rates. In some instances, in 
Table 1: Comparison of the performance of Q-learning for a continuous utility function with a variety of mean models under varying degrees of non-linearity in the confounderoutcome relationship: bias, Monte Carlo variance (MC var), Mean Squared Error (MSE) and coverage of $95 \%$ bootstrap confidence intervals (Cover) of the first interval decision rule parameter $\psi_{10}$. Bias, variance, and MSE are each multiplied by 10 .

\begin{tabular}{lccrrrrrr}
\hline $\begin{array}{l}\text { Adjustment } \\
\text { method }\end{array}$ & Bias & MC var & MSE & Cover & Bias & MC var & MSE & Cover \\
\hline \multicolumn{1}{c}{$f_{1}$ and $f_{2}$ linear } & & & & & & & \\
None & 10.03 & 0.35 & 10.41 & 0.0 & 10.12 & 0.09 & 10.32 & 0.0 \\
Linear & 0.02 & 0.08 & 0.08 & 94.1 & 0.00 & 0.02 & 0.02 & 93.0 \\
PS (linear) & 3.75 & 0.28 & 1.69 & 32.9 & 3.78 & 0.06 & 1.49 & 0.1 \\
PS (quintiles) & 5.17 & 0.27 & 2.94 & 4.3 & 5.14 & 0.07 & 2.71 & 0.0 \\
IPTW & 2.61 & 1.11 & 1.79 & 57.7 & 1.27 & 0.53 & 0.69 & 66.3 \\
GAM & 0.02 & 0.08 & 0.08 & 94.4 & 0.00 & 0.02 & 0.02 & 93.6 \\
$\quad f_{1}$ and $f_{2}$ & quadratic & & & & & & & \\
None & 18.18 & 16.30 & 4.935 & 68.1 & 18.92 & 4.31 & 40.11 & 10.8 \\
Linear & 29.64 & 20.53 & 108.38 & 37.9 & 31.42 & 4.72 & 103.46 & 0.1 \\
PS (linear) & 17.25 & 13.24 & 42.97 & 63.9 & 17.90 & 3.43 & 35.46 & 8.3 \\
PS (quintiles) & 21.10 & 13.85 & 58.35 & 29.4 & 22.42 & 3.53 & 53.81 & 0.2 \\
IPTW & -3.59 & 21.29 & 22.56 & 92.7 & -3.83 & 21.75 & 23.19 & 84.7 \\
GAM & 0.21 & 1.49 & 1.50 & 95.2 & -0.11 & 0.40 & 0.40 & 92.7 \\
$\quad f_{1}$ quadratic, & $f_{2}$ piece-wise polynomial & & & & & \\
None & 7.16 & 15.01 & 20.12 & 91.0 & 6.47 & 4.17 & 8.35 & 82.0 \\
Linear & 32.16 & 21.35 & 124.75 & 35.5 & 33.61 & 5.51 & 118.49 & 0.2 \\
PS (linear) & 17.70 & 14.64 & 45.95 & 64.8 & 17.84 & 3.69 & 35.49 & 10.2 \\
PS (quintiles) & 19.60 & 13.76 & 52.17 & 37.8 & 20.25 & 3.69 & 44.68 & 1.3 \\
IPTW & -5.83 & 30.26 & 33.63 & 90.0 & -4.21 & 20.30 & 22.05 & 81.1 \\
GAM & 0.36 & 2.77 & 2.78 & 92.9 & -0.05 & 0.54 & 0.54 & 95.0 \\
\hline
\end{tabular}

fact, better coverage is achieved by omitting the confounding variables than by including them either as linear terms or via a function of the propensity score, although the mean outcome in a new population is equally poor under all of these approaches (no adjustment, or adjustment by the inclusion of covariates as linear terms or through the PS). When the true functions are not linear, the DTR estimated using the GAM yields mean outcomes that are evidently higher than the outcomes under DTRs estimated using other methods of adjustment (Figure 1, middle and right panel); the DTR estimated by IPTW provides mean outcomes that typically exceed linear or PS methods of adjustment, but also occasionally provide mean outcomes that are worse.

In the discrete outcome setting, we again observed good performance of the estimator resulting from correctly specified Q-functions in the linear relationship settings (panels 1 and 3), and little bias of the estimator from the GAM-based models in the quadratic relationship settings (panels 2 and 4). These results are notable in themselves, as this is the first application of Q-learning to a discrete utility as far as we are aware. Some variations in $\gamma$ and $\zeta$ were considered in addition to the values presented above. The substantive conclusions of the additional simulations were unchanged by such modifications to the data-generation (results not shown). 


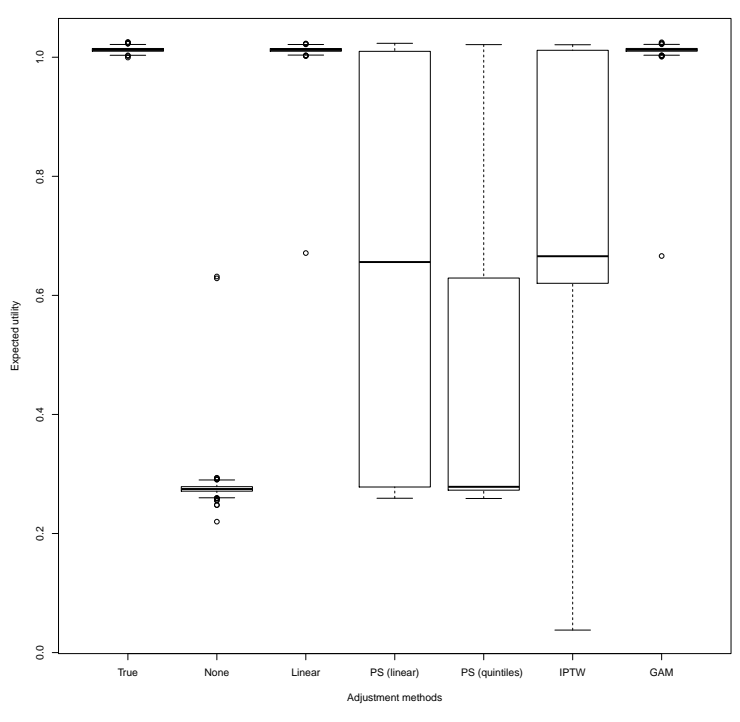

(a) $f_{1}$ and $f_{2}$ linear

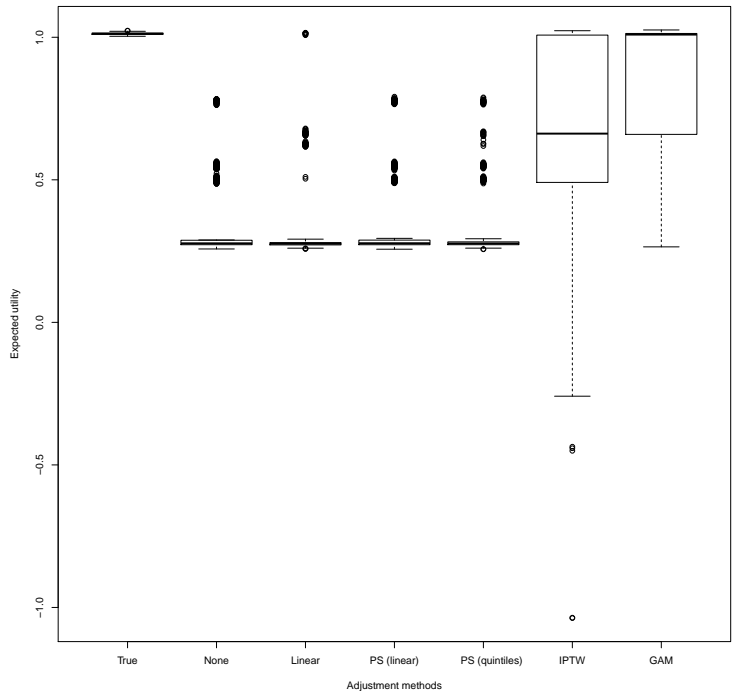

(b) $f_{1}$ and $f_{2}$ quadratic

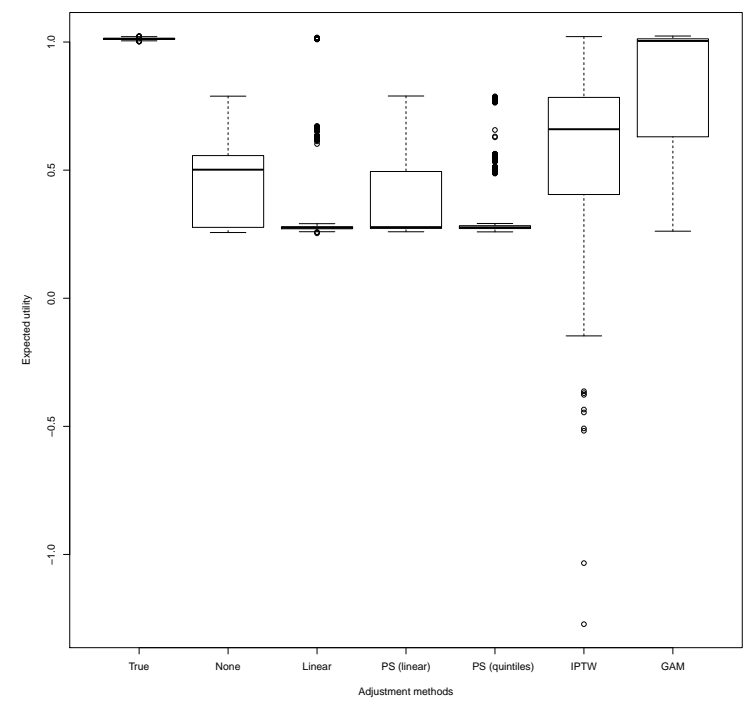

(c) $f_{1}$ quadratic, $f_{2}$ piece-wise polynomial

Figure 1: Mean utility when the true and estimated DTRs are applied to a new population of size 10,000: continuous outcomes. Within each panel, the applied optimal treatment regime is the true optimal regime, and the DTR estimated with no adjustment, linear adjustment, PS (linear) adjustment, PS (quintiles) adjustment, IPTW, and a GAM. 
The Q-learning procedure for a Bernoulli utility under correct linear specification exhibits no bias and coverage at the nominal level for both of the samples sizes considered. When the true model is linear, the GAM specification of the Q-function also yields unbiased estimation and coverage only somewhat higher than the nominal level, at the cost of larger Monte Carlo variability in the smaller sample size. Upon investigation, the large variability can be explained by a very small number (less than $0.5 \%$ ) of cases where the estimate was badly biased despite convergence of the GAM fitting procedure; omitting those estimates from the Monte Carlo variance calculation results in a variance of the Q-function parameters estimated via GAM is approximately $5 \%$, rather than several-fold, larger than the linear model-based estimates.

In the Poisson utility setting, we observe unbiased estimation of the decision regime parameters when the true relationship is linear, using either a GAM or a linear specification of the Q-function with comparable variability in the estimators (Table 2, panel 3), and the DTR estimated using the linear model and the GAM yield mean outcomes that are evidently higher than the outcomes under DTRs estimated using other methods of adjustment (Figure 2, left column). When the relationship between the confounding variable and the outcome is quadratic (Table 2, panel 4), the GAM-based out-performs all other estimators in terms of bias and MSE, however several competing estimators although biased - result in mean outcomes that are comparable to those observed under the GAM-based DTR, and indeed are frequently as good as mean outcomes under the true optimal DTR (Figure 2, right column). We additionally considered the modelling approach of using a GLM at both intervals; this lead to very similar results in terms of bias, variability, and coverage of the estimators, but was computationally much slower. Q-learning using GLMs in both intervals (rather than simply a linear model at the first interval) provided mean outcome distributions much like those from the approach of using a GLM only at the second interval when the true model was linear; in the quadratic case, the expected outcome distribution under the DTR estimated using a linear dependence on the propensity score was skewed much like under the DTR estimated using IPW, exhibiting lower values than outcomes under the DTR estimated by a model that was linear in the covariates or by a GAM.

Based on the results of our simulation study, it can now be seen that Q-learning can be used in a discrete utility setting as well as a continuous utility setting. When the utility is continuous, there appears to be little cost to using a GAM for the Q-function and the approach can therefore be recommended as a first-line approach. For discrete utilities, however, caution may be warranted: while the GAM-based estimators of the decision rule parameters were unbiased under linear and quadratic confounder relationships for both Bernoulli and Poisson utilities, the estimates for binary outcomes in modest samples are occasionally poor. Thus, when the sample size is not large, a careful comparison of the rules implied by a variety of specifications with varying degrees of complexity - for example, one might consider estimators from linear and quadratic models in addition to a GAM - and the associated variability. Whenever sufficient information is available on the longitudinal covariate process, it may also be valuable to simulate outcomes under different estimated regimes; as we observed in some discrete outcome settings, differences in treatment rule parameter estimates may not lead to substantially different outcomes. 
Table 2: Comparison of the performance of Q-learning for a discrete utility function with a variety of mean models under a linear and quadratic confounder-outcome relationship: bias, Monte Carlo variance (MC var), Mean Squared Error (MSE) and coverage of 95\% bootstrap confidence intervals (Cover) of the first interval decision rule parameter $\psi_{10}$. Bias, variance, and MSE are each multiplied by 10 .

\begin{tabular}{|c|c|c|c|c|c|c|c|c|}
\hline \multirow{2}{*}{$\begin{array}{l}\text { Adjustment } \\
\text { method }\end{array}$} & \multicolumn{4}{|c|}{$n=250$} & \multicolumn{4}{|c|}{$n=1000$} \\
\hline & Bias & $\mathrm{MC}$ var & MSE & Cover & Bias & $\mathrm{MC}$ var & MSE & Cover \\
\hline \multicolumn{9}{|c|}{ Bernoulli outcome, $f_{1}$ and $f_{2}$ linear } \\
\hline None & 8.65 & 1.57 & 8.97 & 13.7 & 8.45 & 0.19 & 7.32 & 0.0 \\
\hline Linear & 0.20 & 1.98 & 1.98 & 94.9 & 0.00 & 0.28 & 0.28 & 95.1 \\
\hline PS (linear) & 4.52 & 1.85 & 3.89 & 71.9 & 4.23 & 0.27 & 2.05 & 19.5 \\
\hline PS (quintiles) & 5.02 & 1.80 & 4.31 & 67.0 & 4.76 & 0.24 & 2.50 & 8.3 \\
\hline IPTW & 4.00 & 2.22 & 3.84 & 77.3 & 3.49 & 0.29 & 1.51 & 47.6 \\
\hline GAM & 0.81 & 4.25 & 4.25 & & 0.00 & 0.28 & 0.28 & 95.8 \\
\hline \multicolumn{9}{|c|}{ Bernoulli outcome, $f_{1}$ and $f_{2}$ quadratic } \\
\hline None & 3.77 & 0.65 & 2.07 & 64.8 & 3.71 & 0.15 & 1.53 & 10.8 \\
\hline Linear & 1.54 & 0.87 & 1.11 & 92 & 1.5 & 0.20 & 0.44 & 79.7 \\
\hline PS (linear) & 2.46 & 0.68 & 1.28 & 84. & 2.40 & 0.17 & 0.74 & 47.2 \\
\hline PS (quil & 2.51 & 0.77 & 1.40 & 8 & 2.44 & 0.19 & 0.79 & 51.1 \\
\hline IPTW & 1.92 & 1.02 & 1.39 & 90 & 1.77 & 0.23 & 0.54 & 78.8 \\
\hline GAM & 0.06 & 2.63 & 2.63 & 97.2 & -0.11 & 0.32 & 0.32 & 97.0 \\
\hline \multicolumn{9}{|c|}{ Poisson outcome, $f_{1}$ and $f_{2}$ linear } \\
\hline None & 8.97 & 0.70 & 8.74 & 5.6 & 9.49 & 0.23 & 9.23 & 0.0 \\
\hline Linear & 0.14 & 0.11 & 0.11 & 93.9 & 0.1 & 0.02 & 0.03 & 93.8 \\
\hline PS (line & 3.92 & 0.45 & 1.98 & 43 & 4.1 & 0.14 & 1.85 & 4.1 \\
\hline PS (quintiles) & 5.71 & 0.62 & 3.88 & 29.7 & 6.15 & 0.21 & 3.99 & 0.8 \\
\hline IPTW & 3.16 & 0.85 & 1.85 & 69.3 & 2.5 & 0.33 & 0.96 & 69.1 \\
\hline GAM & 0.13 & 0.11 & 0.11 & 95.7 & 0.14 & 0.02 & 0.03 & 94.5 \\
\hline \multicolumn{9}{|c|}{ Poisson outcome, $f_{1}$ and $f_{2}$ quadratic } \\
\hline None & 4.39 & 0.19 & 2.12 & 15.4 & 4.32 & 0.04 & 1.91 & 0.0 \\
\hline Linear & -1.01 & 0.27 & 0.38 & 90.1 & -1.06 & 0.07 & 0.19 & 72.6 \\
\hline PS (linear) & 1.38 & 0.25 & 0.44 & 87.1 & 1.28 & 0.07 & 0.23 & 63.3 \\
\hline PS (quintiles) & 1.81 & 1.01 & 1.34 & 92.5 & 1.78 & 0.08 & 0.40 & 55.1 \\
\hline IPW & 1.65 & 0.36 & 0.63 & 84.8 & 1.35 & 0.09 & 0.27 & 86.0 \\
\hline GAM & 0.00 & 0.28 & 0.28 & 96.7 & 0.14 & 0.64 & 0.65 & 94.6 \\
\hline
\end{tabular}




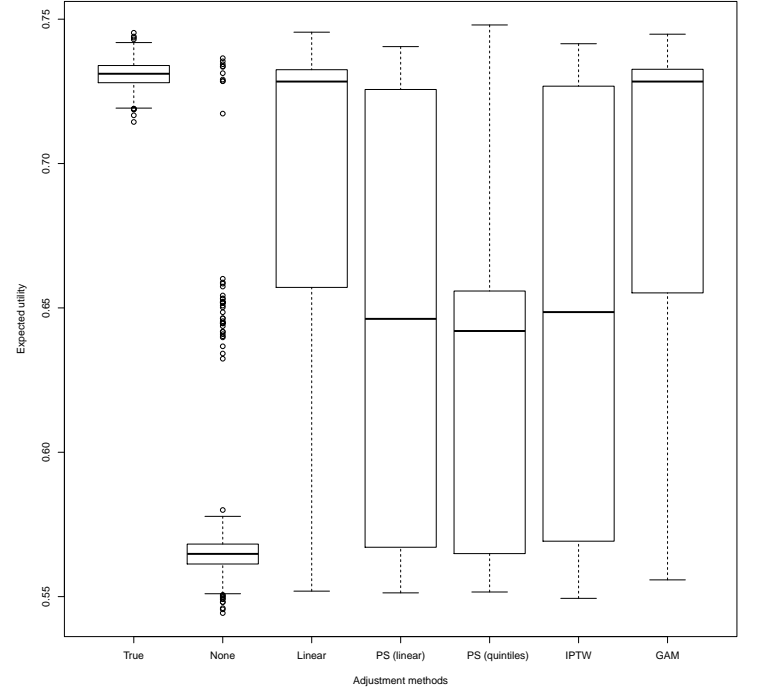

(a) Binary utility, $f_{1}$ and $f_{2}$ linear

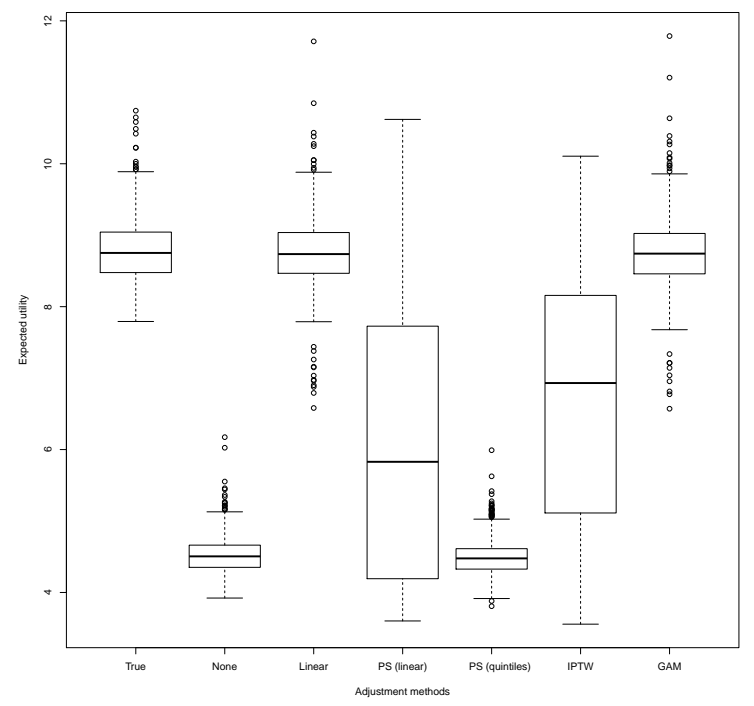

(c) Poisson utility, $f_{1}$ and $f_{2}$ linear

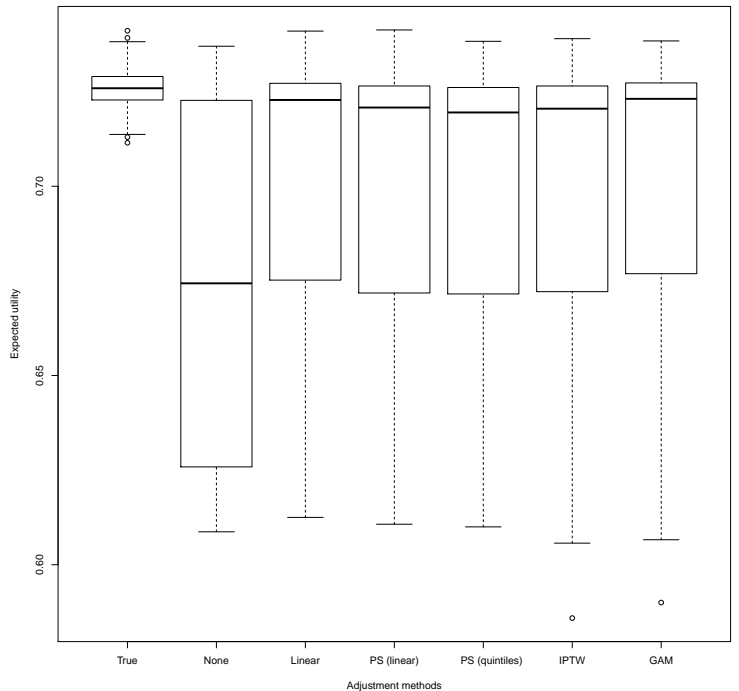

(b) Binary utility, $f_{1}$ and $f_{2}$ quadratic

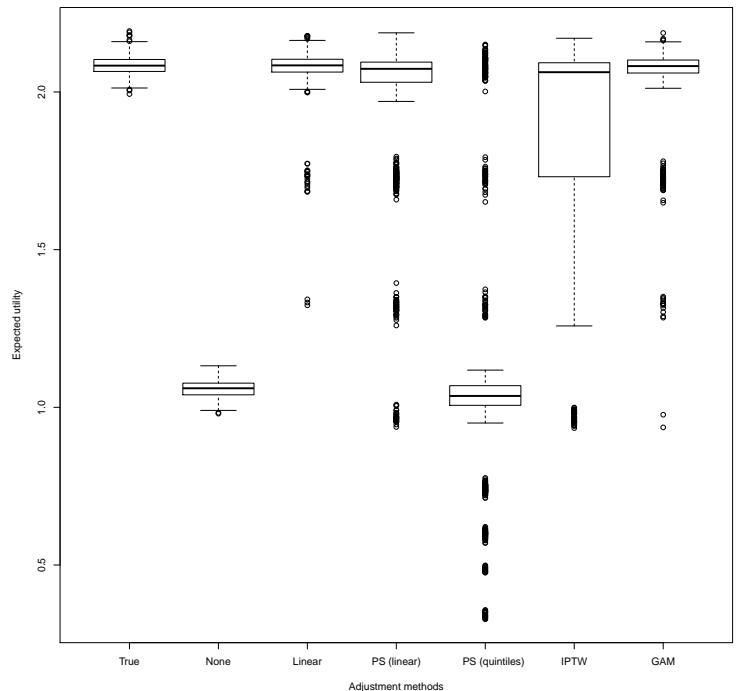

(d) Poisson utility, $f_{1}$ and $f_{2}$ quadratic

Figure 2: Mean utility when the true and estimated DTRs are applied to a new population of size 10,000: discrete outcomes. Within each panel, the applied optimal treatment regime is the true optimal regime, and the DTR estimated with no adjustment, linear adjustment, PS (linear) adjustment, PS (quintiles) adjustment, IPTW, and a GAM. 


\section{Discussion}

In this article, we propose a Q-learning framework for developing optimal dynamic treatment regimes when utilities may be discrete and the dependence of the utility on covariates is complex or unknown. The latter problem is particularly relevant to estimation of dynamic treatment rule parameters from observational data which often present larger sample sizes or represent a more generalizable population, but also carry the potential for the presence of confounding. The generalizations of the typical Q-learning to include the use of generalized additive models for the Q-function with a variety of utility types performs well in simulation, and is a promising step to a more fully general method of estimating optimal dynamic treatment regimes. However, several challenges remain.

In the simpler case of a continuous utility with no sharing of parameters between treatment intervals, it has been shown (4) that Q-learning is equivalent to an inefficient version of g-estimation for parameters of structural nested mean models, the approach proposed by Robins (18) for estimation of dynamic treatment regimes. G-estimation can be applied to discrete outcomes, but the method has not gained wide usage due perhaps to the lack of software packages or libraries available. Thus, while g-estimation is appealing because of its flexibility, Q-learning offers advantages in terms of the ease with which it can be explained to non-statistical researchers who have a familiarity with regression and the very modest coding required for its implementation. It would be of considerable interest to see whether there exists a correspondence between g-estimation and Q-learning in the more complex settings of a discrete-valued utility.

Further generalizing Q-learning with discrete utilities to situations where there are interval-specific rewards that are accumulated is also challenging. Conceptually, it would appear to be a minor and straight-forward modification to the algorithms proposed in Sections 2.2 and 2.3, but in fact it is not quite so simple. For example, if the reward at each interval was binary, the pseudo-outcome in the first interval of a two-interval setting would be the sum of the first-interval reward ( 0 or 1$)$ and the predicted second-interval reward under optimal treatment at the first interval, so that the pseudo-outcome itself would take (continuous) values in the range of $[0,2]$. In the case of a Poisson-valued reward, it is more natural to think of adding or accumulating actual or predicted counts over successive intervals, however deriving the true correspondence between the generative and analytic models is not a trivial undertaking.

Finally, in this article we strove to avoid the issue of non-regularity, assuming in all simulations that there existed a unique optimal treatment decision for all subjects, and that the impact of choosing the correct treatment was relatively large (to avoid nearly nonregular settings where small sample performance of estimators may be erratic). Despite choosing parameters intended to show a large effect, we observed that very different estimates (some biased, others not) could lead to very similar outcome distributions, suggesting that the optimal regime was not unique. In reality - particularly in the setting of randomized trials in which there is an ethical obligation for clinical equipoise on the part of the researchers at the outset of the study - non-regularity is not uncommon. If the null hypothesis of no treatment effect is true, all estimators will be non-regular. In fact, if even a subset of the population is unaffected by treatment so that their utility under the competing treatment alternatives is unchanged, estimators will non-regular. It is therefore of interest to understand the impact of applying a penalization (23) or thresholding (12, 4) to the Q-learning algorithm. A through study of the various bootstrapping procedures (4; 2) that have been proposed as a means of obtaining correct coverage and making 
correct inferences in the presence of non-regularity should also be undertaken.

\section{Acknowledgements}

We would like to thank Dr. Bibhas Chakraborty for insightful discussions. This work is supported by Dr. Moodie's Discovery Grant from the Canada's Natural Sciences and Engineering Research Council (NSERC).

\section{References}

[1] B. Chakraborty. Dynamic treatment regimes for managing chronic health conditions: A statistical perspective. American Journal of Public Health, 101(1):40 - 45, 2011.

[2] B. Chakraborty, E.B. Laber, and Y. Zhao. Inference for optimal dynamic treatment regimes using an adaptive m-out-of-n bootstrap scheme. 2013. Submitted.

[3] B. Chakraborty and E.E.M. Moodie. Estimating optimal dynamic treatment regimes with shared decision rules across stages: An extension of Q-learning. 2013. Submitted.

[4] B. Chakraborty, S.A. Murphy, and V. Strecher. Inference for non-regular parameters in optimal dynamic treatment regimes. Statistical Methods in Medical Research, 19(3):317 - 343, 2010.

[5] M. Fava, A.J. Rush, M.H. Trivedi, A.A. Nierenberg, M.E. Thase, H.A. Sackeim, F.M. Quitkin, S. Wisniewski, P.W. Lavori, J.F. Rosenbaum, and D.J. Kupfer. Background and rationale for the sequenced treatment alternatives to relieve depression (STAR*D) study. Psychiatric Clinics of North America, 26(2):457 - 494, 2003.

[6] G. Golub, M. Heath, and G. Wahba. Generalized cross validation as a method for choosing a good ridge parameter. Technometrics, 21:215 - 224, 1979.

[7] Trevor Hasite and Robert Tibshirani. Generalized additive models. Statistical Science, 1(3):297-318, 1986.

[8] Trevor Hasite and Robert Tibshirani. Generalized Additive Models. Chapman \& Hall/CRC, 1990.

[9] X. Huang and J. Ning. Analysis of multi-stage treatments for recurrent diseases. Statistics in Medicine, 31:2805 - 2821, 2012.

[10] K.C. Li. Asymptotic optimality of $C_{p}, C_{L}$, cross-validation and generalized crossvalidation: Discrete index set. Annals of Statistics, 15:958 -975, 1987.

[11] E.E.M. Moodie, B. Chakraborty, and M.S. Kramer. Q-learning for estimating optimal dynamic treatment rules from observational data. Canadian Journal of Statistics, 40:629 - 645, 2012.

[12] E.E.M. Moodie and T.S. Richardson. Estimating optimal dynamic regimes: correcting bias under the null. Scandinavian Journal of Statistics, 37:126-146, 2010. 
[13] S. A. Murphy, D. W. Oslin, A. J. Rush, and J. Zhu. Methodological challenges in constructing effective treatment sequences for chronic psychiatric disorders. Neuropsychopharmacology, 32:257 -262, 2007.

[14] S.A. Murphy. A generalization error for Q-learning. Journal of Machine Learning Research, 6:1073-1097, 2005.

[15] I Nahum-Shani, M Qian, D Almirall, W E Pelham, B Gnagy, G A Fabiano, J G Waxmonsky, J Yu, and S A Murphy. Q-learning: A data analysis method for constructing adaptive interventions. Psychological Methods, 17:478 - 494, 2012.

[16] R Core Team. R: A Language and Environment for Statistical Computing. R Foundation for Statistical Computing, Vienna, Austria, 2012. ISBN 3-900051-07-0.

[17] J M Robins, M A Hernán, and B Brumback. Marginal structural models and causal inference in epidemiology. Epidemiology, 11:550-60, 2000.

[18] J.M. Robins. Optimal structural nested models for optimal sequential decisions. In D.Y. Lin and P. Heagerty, editors, Proceedings of the Second Seattle Symposium on Biostatistics, pages 189-326, New York, 2004. Springer.

[19] P.R. Rosenbaum and D.B. Rubin. The central role of the propensity score in observational studies for causal effects. Biometrika, 70:41 - 55, 1983.

[20] S. Rosthoj, C. Fullwood, R. Henderson, and S. Stewart. Estimation of optimal dynamic anticoagulation regimes from observational data: a regret-based approach. Statistics in Medicine, 25:4197-4215, 2006.

[21] L.S. Schneider, P.N. Tariot, C.G. Lyketsos, K.S. Dagerman, K.L. Davis, and S. Davis. National institute of mental health clinical antipsychotic trials of intervention effectiveness (CATIE): Alzheimer disease trial methodology. American Journal of Geriatric Psychology, 9:346-360, 2001.

[22] S.M. Shortreed and E.E.M. Moodie. Estimating the optimal dynamic antipsychotic treatment regime: Evidence from the sequential-multiple assignment randomized CATIE schizophrenia study. Journal of the Royal Statistical Society, Series B, 61:577-599, 2012.

[23] R. Song, W. Wang, D. Zeng, and M.R. Kosorok. Penalized q-learning for dynamic treatment regimes. 2013. Submitted.

[24] R.S. Sutton and A.G. Barto. Reinforcement learning: An introduction. MIT Press, Cambridge, 1998.

[25] P.F. Thall, R.E. Millikan, and H.G. Sung. Evaluating multiple treatment courses in clinical trials. Statistics in Medicine, 30:1011-1128, 2000.

[26] P.F. Thall, H.G. Sung, and E.H. Estey. Selecting therapeutic strategies based on efficacy and death in multicourse clinical trials. Journal of the American Statistical Association, 97(457):29-39, 2002.

[27] E. Topol. Creative Destruction of Medicine: How the Digital Revolution and Personalized Medicine Will Create Better Health Care. Basic Books, New York, 2012. 
[28] Simon N. Wood. Stable and efficient multiple smoothing parameter estimation for generalized additive models. Journal of the American Statistical Association, 99(467):673-686, 2004.

[29] Simon N. Wood. Generalized Additive Models: An Introduction with R. Chapman \& Hall/CRC, 2006.

[30] Simon N. Wood. Fast stable restricted maximum likelihood and marginal likelihood estimation of semiparametric generalized linear models. Journal of the Royal Statistical Society (B), 73(1):3-36, 2011.

[31] Jingyi Xin, Bibhas Chakraborty, and Eric B. Laber. qLearn: Estimation and inference for Q-learning, 2012. R package version 1.0.

[32] Y. Zhao, M.R. Kosorok, and D. Zeng. Reinforcement learning design for cancer clinical trials. Statistics in Medicine, 28:3294 - 3315, 2009.

[33] Y. Zhao, D. Zeng, M.A. Socinski, and M.R. Kosorok. Reinforcement learning strategies for clinical trials in nonsmall cell lung cancer. Biometrics, 67(4):1422 - 1433, 2011.

\section{Appendix. Derivation of the True Dynamic Regime Parameters}

In the following, we calculate the true values of the first-interval decision rule parameters $\psi_{10}$ and $\psi_{11}$ in terms of $\gamma^{\prime}$ 's and $\delta$ 's, the parameters of the generative model following the calculations in [2].

\section{A.1 Bernoulli utility}

We begin with the derivations for the case of a Bernoulli utility. Let $M=\gamma_{0}+\gamma_{1} C_{1}+$ $\gamma_{2} O_{1}+\gamma_{3} A_{1}+\gamma_{4} O_{1} A_{1}+\gamma_{5} C_{2}+f_{1}\left(C_{1}\right)+f_{2}\left(C_{2}\right)$, and define $\mu=M+\gamma_{6} A_{2}+\gamma_{7} O_{2} A_{2}+\gamma_{8} A_{1} A_{2}$. It follows that

$$
\begin{aligned}
& \max _{a_{2}} Q_{2}\left(H_{2}, a_{2}\right)= \operatorname{expit}\left(\mathrm{M}+\left|\gamma_{6}+\gamma_{7} \mathrm{O}_{2}+\gamma_{8} \mathrm{~A}_{1}\right|\right) \\
&=\operatorname{expit}\left(\mathrm{M}+\frac{1}{4}\left(1+\mathrm{O}_{2}\right)\left(1+\mathrm{A}_{1}\right)\left|\mathrm{f}_{1}\right|+\frac{1}{4}\left(1+\mathrm{O}_{2}\right)\left(1-\mathrm{A}_{1}\right)\left|\mathrm{f}_{2}\right|\right. \\
&\left.\quad+\frac{1}{4}\left(1-O_{2}\right)\left(1+A_{1}\right)\left|f_{3}\right|+\frac{1}{4}\left(1-O_{2}\right)\left(1-A_{1}\right)\left|f_{4}\right|\right)
\end{aligned}
$$

where $f_{1}=\gamma_{6}+\gamma_{7}+\gamma_{8}, f_{2}=\gamma_{6}+\gamma_{7}-\gamma_{8}, f_{3}=\gamma_{6}-\gamma_{7}+\gamma_{8}$, and $f_{4}=\gamma_{6}-\gamma_{7}-\gamma_{8}$. Further,

$$
\begin{aligned}
E\left(O_{2} \mid O_{1}, A_{1}\right) & =\frac{\exp \left(\delta_{1} O_{1}+\delta_{2} A_{1}\right)-1}{\exp \left(\delta_{1} O_{1}+\delta_{2} A_{1}\right)+1} \\
1+E\left(O_{2} \mid O_{1}, A_{1}\right) & =2 \operatorname{expit}\left(\delta_{1} \mathrm{O}_{1}+\delta_{2} \mathrm{~A}_{1}\right), \\
1-E\left(O_{2} \mid O_{1}, A_{1}\right) & =2\left(1-\operatorname{expit}\left(\delta_{1} \mathrm{O}_{1}+\delta_{2} \mathrm{~A}_{1}\right)\right) .
\end{aligned}
$$


Therefore,

$$
\begin{aligned}
Q_{1}\left(H_{1}, A_{1}\right)= & E\left[\max _{a_{2}} Q_{2}\left(H_{2}, a_{2}\right) \mid H_{1}, A_{1}\right] \\
= & \operatorname{expit}\left(\mathrm{M}+\frac{1}{2}\left(1+\mathrm{A}_{1}\right)\left|\mathrm{f}_{3}\right|+\frac{1}{2}\left(1-\mathrm{A}_{1}\right)\left|\mathrm{f}_{4}\right|\right. \\
& +\frac{1}{2} \operatorname{expit}\left(\delta_{1} \mathrm{O}_{1}+\delta_{2} \mathrm{~A}_{1}\right)\left(1+\mathrm{A}_{1}\right)\left(\left|\mathrm{f}_{1}\right|-\left|\mathrm{f}_{3}\right|\right) \\
& \left.+\frac{1}{2} \operatorname{expit}\left(\delta_{1} \mathrm{O}_{1}+\delta_{2} \mathrm{~A}_{1}\right)\left(1-\mathrm{A}_{1}\right)\left(\left|\mathrm{f}_{2}\right|-\left|\mathrm{f}_{4}\right|\right)\right) .
\end{aligned}
$$

Furthermore,

$$
\begin{aligned}
4 \operatorname{expit}\left(\delta_{1} \mathrm{O}_{1}+\delta_{2} \mathrm{~A}_{1}\right)=(1 & \left.+O_{1}\right)\left(1+A_{1}\right) \operatorname{expit}\left(\delta_{1}+\delta_{2}\right) \\
& +\left(1+O_{1}\right)\left(1-A_{1}\right) \operatorname{expit}\left(\delta_{1}-\delta_{2}\right) \\
& +\left(1-O_{1}\right)\left(1+A_{1}\right) \operatorname{expit}\left(-\delta_{1}+\delta_{2}\right) \\
& +\left(1-O_{1}\right)\left(1-A_{1}\right) \operatorname{expit}\left(-\delta_{1}-\delta_{2}\right) .
\end{aligned}
$$

Since $A_{1} \in\{-1,1\}$, we have $\left(1-A_{1}^{2}\right)=0,\left(1+A_{1}\right)^{2}=2\left(1+A_{1}\right)$ and $\left(1-A_{1}\right)^{2}=2\left(1-A_{1}\right)$. It may therefore be deduced that

$$
\begin{aligned}
2 \operatorname{expit}\left(\delta_{1} \mathrm{O}_{1}+\delta_{2} \mathrm{~A}_{1}\right)\left(1+\mathrm{A}_{1}\right)=(1 & \left.+O_{1}\right)\left(1+A_{1}\right) \operatorname{expit}\left(\delta_{1}+\delta_{2}\right) \\
& +\left(1-O_{1}\right)\left(1+A_{1}\right) \operatorname{expit}\left(-\delta_{1}+\delta_{2}\right)
\end{aligned}
$$

and

$$
\begin{aligned}
\operatorname{expit}\left(\delta_{1} \mathrm{O}_{1}+\delta_{2} \mathrm{~A}_{1}\right)\left(1-\mathrm{A}_{1}\right)=(1 & \left.+O_{1}\right)\left(1-A_{1}\right) \operatorname{expit}\left(\delta_{1}-\delta_{2}\right) \\
& +\left(1-O_{1}\right)\left(1-A_{1}\right) \operatorname{expit}\left(-\delta_{1}-\delta_{2}\right) .
\end{aligned}
$$

Let $k_{1}=\frac{1}{4} \operatorname{expit}\left(\delta_{1}+\delta_{2}\right), \mathrm{k}_{2}=\frac{1}{4} \operatorname{expit}\left(-\delta_{1}+\delta_{2}\right), \mathrm{k}_{3}=\frac{1}{4} \operatorname{expit}\left(\delta_{1}-\delta_{2}\right), \mathrm{k}_{4}=\frac{1}{4} \operatorname{expit}\left(-\delta_{1}-\right.$ $\left.\delta_{2}\right)$. Therefore,

$$
\begin{aligned}
Q_{1}\left(H_{1}, A_{1}\right)= & \operatorname{expit}\left(\mathrm{M}+\frac{1}{2}\left(\left|\mathrm{f}_{3}\right|+\left|\mathrm{f}_{4}\right|\right)+\frac{1}{2}\left(\left|\mathrm{f}_{3}\right|-\left|\mathrm{f}_{4}\right|\right) \mathrm{A}_{1}\right. \\
& +\left(1+O_{1}\right)\left(1+A_{1}\right) k_{1}\left(\left|f_{1}\right|-\left|f_{3}\right|\right)+\left(1-O_{1}\right)\left(1+A_{1}\right) k_{2}\left(\left|f_{1}\right|-\left|f_{3}\right|\right) \\
& \left.+\left(1+O_{1}\right)\left(1-A_{1}\right) k_{3}\left(\left|f_{2}\right|-\left|f_{4}\right|\right)+\left(1-O_{1}\right)\left(1-A_{1}\right) k_{4}\left(\left|f_{2}\right|-\left|f_{4}\right|\right)\right) .
\end{aligned}
$$

Applying a logit transformation to $Q_{1}\left(H_{1}, A_{1}\right)$ gives that the coefficient of $A_{1}$ in the above expression for $\operatorname{logit}\left(\mathrm{Q}_{1}\left(\mathrm{H}_{1}, \mathrm{~A}_{1}\right)\right)$ is:

$$
\psi_{10}=\gamma_{3}+\left(k_{1}+k_{2}\right)\left|f_{1}\right|-\left(k_{3}+k_{4}\right)\left|f_{2}\right|+\left(k_{3}+k_{4}\right)\left|f_{3}\right|-\left(k_{1}+k_{2}\right)\left|f_{4}\right|,
$$

and the coefficient of $O_{1} A_{1}$ in the expression for $Q_{1}$ is:

$$
\psi_{11}=\gamma_{4}+\left(k_{1}-k_{2}\right)\left|f_{1}\right|-\left(k_{3}-k_{4}\right)\left|f_{2}\right|-\left(k_{1}-k_{2}\right)\left|f_{3}\right|+\left(k_{3}-k_{4}\right)\left|f_{4}\right| .
$$




\section{A.2 Poisson utility}

In this section, we derive the correspondence between the Q-function model parameters and the parameters from the data-generating models when the utility is given by a Poisson count. As above, let $M=\gamma_{0}+\gamma_{1} C_{1}+\gamma_{2} O_{1}+\gamma_{3} A_{1}+\gamma_{4} O_{1} A_{1}+\gamma_{5} C_{2}+f_{1}\left(C_{1}\right)+f_{2}\left(C_{2}\right)$, and $\mu=M+\gamma_{6} A_{2}+\gamma_{7} O_{2} A_{2}+\gamma_{8} A_{1} A_{2}$. Then

$$
\begin{aligned}
\max _{a_{2}} Q_{2}\left(H_{2}, a_{2}\right)=\exp & \left(M+\frac{1}{4}\left(1+O_{2}\right)\left(1+A_{1}\right)\left|f_{1}\right|+\frac{1}{4}\left(1+O_{2}\right)\left(1-A_{1}\right)\left|f_{2}\right|\right. \\
+ & \left.\frac{1}{4}\left(1-O_{2}\right)\left(1+A_{1}\right)\left|f_{3}\right|+\frac{1}{4}\left(1-O_{2}\right)\left(1-A_{1}\right)\left|f_{4}\right|\right)
\end{aligned}
$$

where $f_{1}, f_{2}, f_{3}$, and $f_{4}$ are as defined above. Thus,

$$
\begin{aligned}
Q_{1}\left(H_{1}, A_{1}\right)= & \exp \left(M+\frac{1}{2}\left(1+A_{1}\right)\left|f_{3}\right|+\frac{1}{2}\left(1-A_{1}\right)\left|f_{4}\right|\right. \\
& +\frac{1}{2} \operatorname{expit}\left(\delta_{1} \mathrm{O}_{1}+\delta_{2} \mathrm{~A}_{1}\right)\left(1+\mathrm{A}_{1}\right)\left(\left|\mathrm{f}_{1}\right|-\left|\mathrm{f}_{3}\right|\right) \\
& \left.+\frac{1}{2} \operatorname{expit}\left(\delta_{1} \mathrm{O}_{1}+\delta_{2} \mathrm{~A}_{1}\right)\left(1-\mathrm{A}_{1}\right)\left(\left|\mathrm{f}_{2}\right|-\left|\mathrm{f}_{4}\right|\right)\right) \\
= & \exp \left(M+\frac{1}{2}\left(\left|f_{3}\right|+\left|f_{4}\right|\right)+\frac{1}{2}\left(\left|f_{3}\right|-\left|f_{4}\right|\right) A_{1}\right. \\
& +\left(1+O_{1}\right)\left(1+A_{1}\right) k_{1}\left(\left|f_{1}\right|-\left|f_{3}\right|\right)+\left(1-O_{1}\right)\left(1+A_{1}\right) k_{2}\left(\left|f_{1}\right|-\left|f_{3}\right|\right) \\
& \left.+\left(1+O_{1}\right)\left(1-A_{1}\right) k_{3}\left(\left|f_{2}\right|-\left|f_{4}\right|\right)+\left(1-O_{1}\right)\left(1-A_{1}\right) k_{4}\left(\left|f_{2}\right|-\left|f_{4}\right|\right)\right)
\end{aligned}
$$

where $k_{1}, k_{2}, k_{3}, k_{4}$ are as above. Therefore, the coefficients of $A_{1}$ and $O_{1} A_{1}$ in the above expression take the same form as in the case of a Bernoulli utility:

$$
\begin{aligned}
& \psi_{10}=\gamma_{3}+\left(k_{1}+k_{2}\right)\left|f_{1}\right|-\left(k_{3}+k_{4}\right)\left|f_{2}\right|+\left(k_{3}+k_{4}\right)\left|f_{3}\right|-\left(k_{1}+k_{2}\right)\left|f_{4}\right|, \\
& \psi_{11}=\gamma_{4}+\left(k_{1}-k_{2}\right)\left|f_{1}\right|-\left(k_{3}-k_{4}\right)\left|f_{2}\right|-\left(k_{1}-k_{2}\right)\left|f_{3}\right|+\left(k_{3}-k_{4}\right)\left|f_{4}\right| .
\end{aligned}
$$

